\title{
Intelligent Multiagent Approach to Morbidity by Ixodes Tick- Born Borreliosis Forecasting
}

\author{
Dmytro Chumachenko ${ }^{1}$, Pavlo Piletskiy ${ }^{2}$, Mariia Sukhorukova ${ }^{3}$ \\ ${ }^{I}$ PhD, Assosiate Professor of Informatics Department, National Aerospace University "Kharkiv Aviation \\ Institute", Kharkiv, Ukraine \\ ${ }^{2}$ Bachelor, Informatics Department, National Aerospace University "Kharkiv Aviation Institute", Kharkiv, \\ Ukraine \\ ${ }^{3}$ Teaching Assistant, Epidemiology Department, Kharkiv National Medical University, Kharkiv, Ukraine \\ *Corresponding Author: Dmytro Chumachenko, Ukraine, Kharkiv, 23 Serpnya str., 18, fl.Ukraine, \\ Email: dichumachenko@gmail.com
}

\begin{abstract}
The aim of the study is to construct an intelligent multiagent model of the dynamics of the spread of morbidity by Ixodes tick borreliosis. The proposed model allows taking into account heterogeneity of the population, knowledge base of agents, as well as intellectual communications of the modeled population. The intelligent multiagent approach to simulation allows increasing the reliability of the model. This will allow epidemiological experts to take timely and effective anti-epidemic measures to reduce morbidity, which has a high social and economic importance for society
\end{abstract}

Keywords: Epidemic process, simulation of epidemic process, Ixodes tick borreliosis, Lyme disease, multiagent simulation, agent-based simulation

\section{INTRODUCTION}

Climate warming, globalization, social and economic crises lead to the activation of natural foci of vector-borne infections, among which a special place belongs to Ixodes tick-born borreliosis (Lyme disease), the etiological factors of which are the borrelies of group B. Burgdorferi, and the carrier - Ixodes ticks [1]. Clinically, the disease occurs with a predominant lesion of the skin, nervous system, musculoskeletal system, heart muscle, characterized by a tendency to chronic, as well as latent flow [2].

More than 5,000 cases are registered in the United States every year. In European countries, according to German scientists, the number of cases can be up to 8-10 thousand per year. Intensive morbidity rates for borreliosis in France are 39.4 per 100 thousand populations, in Bulgaria - 36.6 [3].

In Ukraine, according to the data of the Lviv Research Institute of Epidemiology and Hygiene, the quantity of ticks infected by borrelias is 10$70 \%$ among all, from $10 \%$ to $42.2 \%$ of Ukraine's population had contact with the causative agent of borreliosis. Because of incomplete and untimely detection of diseases of this group, it is impossible to assess the actual state of the disease, which makes them epidemiologically uncontrolled and poses a significant threat to the occurrence of epidemic complications. Identifying the most significant risk factors and predicting the incidence of Ixodes tick borreliosis will allow selecting the most effective preventive measures, preventing complications of the epidemiological situation, increasing the number of acute and chronic cases of the disease [4].

The aim of the research is to predict the dynamics of epidemic process of Ixodes tick borreliosis in given area.

\section{Materials And Methods}

The multiagent approach to simulation of epidemic process has been used. Agent technologies are associated with the concept of an intelligent agent, as some robot, purposefully interacting with other similar elements and the external environment under given conditions.

An intelligent agent is an imitation model of an active element, the state and behavior of which in various situations of achieving the goal vary depending on the state and behavior of other agents and the environment, in analogy with the 
intellectual behavior of a live organism (including a human) under similar conditions.

Thus, agent technologies are associated with the simulation of the interaction of intelligent agents - active elements of dynamic systems of any physical, biological and social nature. Behavior and change in the state of such systems are the result of a step-by-step interaction of many of its active elements, the nature of the relations and relationships between them, the conditions for achieving local and global goals, etc. In this case, formalization and modeling of the processes of agent behavior and interaction allow imitating and predicting the emergence of qualitatively new states of the system and to assess the possibility of achieving the goal with different alternatives, as well as to justify making decisions in complex risk situations, uncertainty and conflict.

\subsection{Analysis of Existing Epidemic Process Models}

In the healthcare and epidemiology, itself, modeling is used to quantify the benefits of various methods of control and prevention, such as isolation and restriction measures, vaccination and selection of contingents for immunization, identification of risk groups for morbidity, etc., which is necessary for health authorities to make the most rational and effective measures to fight infections. Only correctly formulated mathematical models allow us to approach a rigorous study of all aspects of the problem, regardless of whether it is an epidemiological diagnosis, an assessment of the effectiveness of existing preventive and antiepidemic measures, or about activities planned by the health authorities and the sanitary and epidemiological service [5].

To select the optimal method for modeling the spread of morbidity, let's consider the most popular and practically significant of the already existing models.

Daniel Bernoulli presented a paper on the analysis of mortality from smallpox. In it, using the simplest mathematical apparatus based on differential equations, he estimated the effectiveness of preventive measures. The work of Bernoulli can be considered the first model for the spread of morbidity.

The next stage in the development of the epidemic process modeling was the work of William Farr. He studied and modeled the statistical mortality of the population of England (Wales) from the smallpox epidemic in 18371839 and obtained mathematical models of indicators of the "movement" of the epidemic of smallpox in the form of statistical regularities. The obtained models allowed him to make a prognostic model of this epidemic. At the beginning of the 20th century, Farr's statistical approach to the study of epidemics was rethought and then developed in the works of John Brownlee [6].

The most famous among these models are the SIR and SEIR models. The SIR model was proposed by Kermak and McKendrick in 1927. In this model, the entire population on the simulated territory is divided into groups: "Susceptible" - people susceptible to a certain impact (S); "Infectious" - people, under the influence of a certain process $(I)$; "Recovered" people who have been exposed to certain effects, more to him are not receptive $(R)$ [7]. Given that the total number of individuals in the population remains unchanged, the increment in the number of people in each of the selected groups can be described using the following system of equations:

$\frac{d S}{d t}=-\beta S I, \frac{d I}{d t}=\beta S I-\gamma I, \frac{d R}{d t}=\gamma I$,

where $\beta$ intensity of contacts between individuals, $\gamma$ is intensity of transferring of individuals to state $R$.

There are modifications of the SIR model, designed to take into account the characteristics of one or another simulated process. In most cases, they are used to simulate the dynamics of the epidemic process of morbidity. For example, the model SEIR is suitable for modeling the spread of influenza. In it to the above-mentioned groups of modeled individuals of the SIR model one more state is added: "Exposed" - people whose disease is in the incubation period $(E)$ [8]. Then the system of equations describing the increment of the number of sick individuals will be:

$$
\begin{aligned}
& \frac{d S}{d t}=B-\beta S I-\mu S, \frac{d E}{d t}=\beta S I-(\varepsilon+\mu) E, \frac{d I}{d t}= \\
& \varepsilon E-(\gamma+\mu) I, \frac{d R}{d t}=\gamma I-\mu R,
\end{aligned}
$$

where $B$ is average birth rate of individuals at simulated area, $\mu$ is average death rate of 
individuals at simulated area, $1 / \varepsilon$ is average length of disease incubation period.

The greatest contribution to the development of the simulation of the epidemic process in recent years has been made by population models. Population models are discrete-event models in which all simulated individuals are clearly divided into social groups that are formed taking into account the age of individuals, in detailed models the occupation of the individual can be taken into account. The spread of infection between individuals can occur only within the framework of one "contact" group. Every day in the model, individuals, depending on their social group, form certain contact groups in which a sick individual can be transmitted to a healthy individual [9]. Contact groups are determined by the characteristic structure of society, which will depend on the modeled territory.

\subsection{Multiagent model of Ixodes tick borreliosis epidemic process}

Mathematical modeling as an element of monitoring of natural focal infections makes it possible to assess the epidemiological potential of foci in the region and in individual territories, to forecast the trends of the epidemic process and to determine the main priorities and directions in the prevention of Ixodes tick borreliosis. Predicting the spread of this disease will allow to establish the main factors influencing the intensification of the epidemic process of the Ixodes tick borreliosis, and to conduct rational preventive and antiepidemic measures with minimal financial and labor costs.

In this research, the process of developing the forecast is carried out using simulation multiagent approach.

A fundamental difference between the new concept of modeling is the introduction and formalization of sensory connections (variables) between the interacting active elements of the dynamic system. These relationships determine the change in the state and behavior of interacting agents and the system as a whole in the direction of "survival" and achievement of goals in complex situations of agreement and opposition, initial uncertainty, risk and conflict, incomplete and unclear information about the degree of achievement of the goal.

An agent is a software module capable of performing the functions assigned to it by some living or cybernetic organism depending on the functions of another agent and the effects of the active medium.

In accordance with the level of artificial intelligence and way of behavior, agents can be classified into the following main types:

- Reflexive agents characterized by physical and social states; have a simple behavior in the form of reactions to current environmental changes and information from other agents on the "condition-action" product rules;

- Knowledge-oriented agents have a physical, social and cognitive state; their behavior is based on a priori knowledge of the environment, identifying the situation and making a decision to achieve the goal;

- Purposeful trained intellectual agents have a given knowledge base and hierarchy of goals, a bank of behavior models and strategies to achieve the goal in conditions of uncertainty, risk and opposition;

- Self-learning, purposeful agents are able to accumulate knowledge on the basis of a large amount of data and ontology of events in the process of interaction with other agents and the environment, adapt to the situation, choose a strategy for achieving the chosen goal and assess the degree of its achievement;

- Emotionally-motivated agents possess, along with the above-described "abilities" of the preceding classes, the emotional state and psychotype in the models of human behavior.

The behavior of the agent is described as an iterative procedure for processing data on the state of other agents and the environment with the choice of a strategy for targeted actions, and is represented by a sequence of operations in discrete time periods, called timely events.

Each operation corresponds to its algorithmic and its program modules, which provide:

- perception of information and the accumulation of knowledge about the environment and the environment of interaction or conflict (sensory module);

- mechanism of interaction and data processing from counterparties;

- analysis of one's own state and the status of counterparties with the selection or correction of target functions (intelligent module);

- making autonomous decisions and choosing strategies. The behavior of the agent can be represented by some recursive form that 
describes the finding and selection at the next step of the transition function from the initial state to the new state in the direction of improving the objective function.

\subsection{Program Realization of Ixodes Tick Borreliosis Multiagent Model}

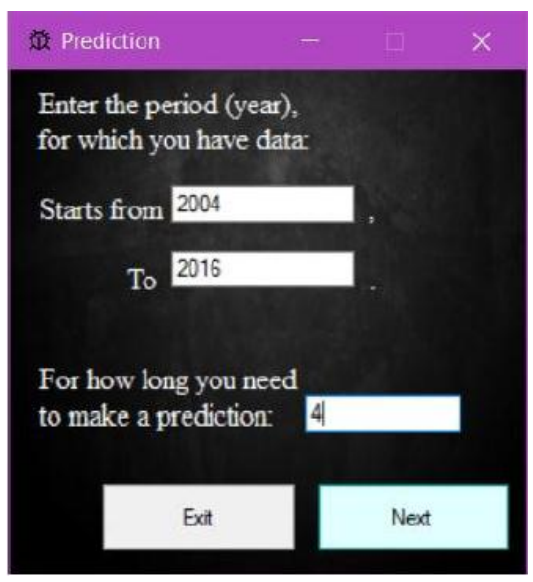

Figure1. Initial window of the program

To automate the prediction of the incidence of Ixodes tick borreliosis a software package has been developed using C\# programing language, that allows calculating prognosis morbidity based on existing statistical data in real time. In developed model, the configuration of the software package includes data for the period 2006 - 2017. The data for the years include intensive incidence rates per 100,000 population, the population's negotiability for tick bites, the results of studies of ticks removed from humans, the presence of Borrelia and the proportion of ticks infected by Borrelia collected by the flagging method, the numbers of ticks.

To start calculating the forecast, you must enter the years for which data are available, and the number of years for which you need to perform a forecast (Fig. 1).

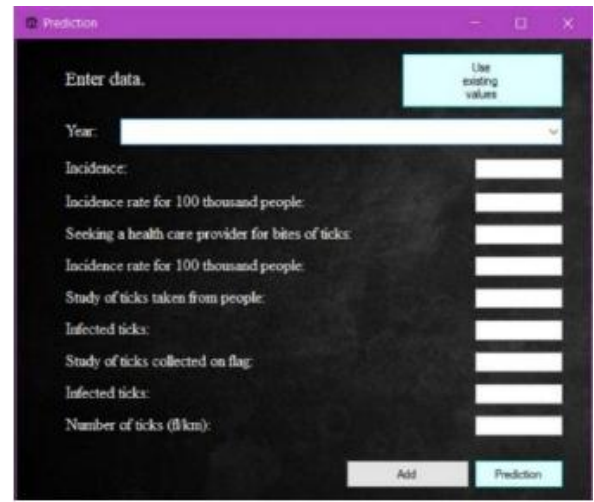

Figure2. Choice of the year for forecasting

Then you need to enter the data for each year or select the available values from the database and perform a forecast (Fig. 2).
After entering the data for one year, you need to click on the "Add" button. After filling all the values for each year, you must click "Prediction" to complete the forecast (Fig. 3).

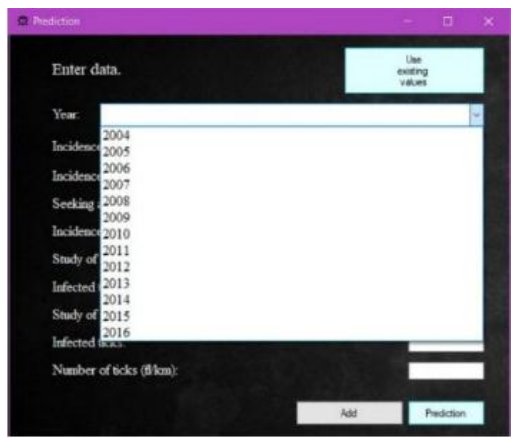

Figure3. Entering the data for forecasting

The program complex automatically calculates the forecast, the results of which are displayed in the form of graphs. Pointing the mouse cursor at the point of interest you can see the exact value.

\section{RESUlTS OF FORECASTING}

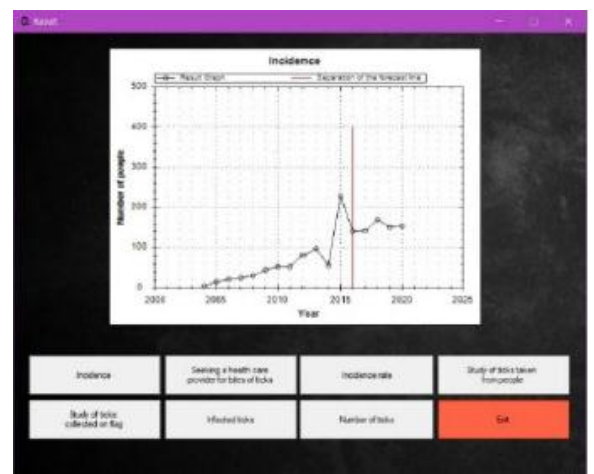

Figure4. Dynamics of incidence rates of Lyme disease.

The calculated prognosis (Figure 4) shows the expected increase in the number of cases with a certain three-year cycle: an increase in the number of cases of Ixodes tick borreliosis within 2 years with a slight decrease for the next year.

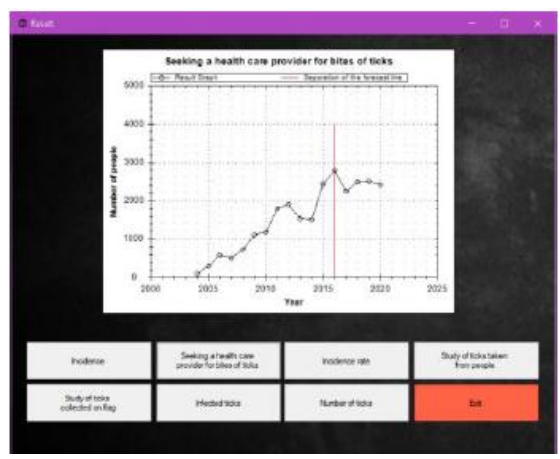

Figure5. Indicators of the incidence of Lyme disease and the number of people seeking medical help for tick bites in terms of 100 thousand people.

As can be seen from the graph (Fig. 5), general trends in the incidence of Lyme disease 
morbidity and the population's request for medical assistance for ticks by 100,000 people are projected.

Based on the calculated prognosis (Fig. 6), a slight decrease and stabilization of the number of ticks in natural habitats can be expected.

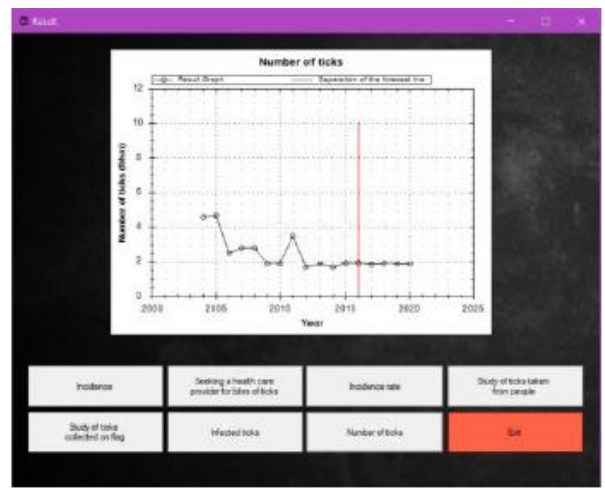

Figure6. The indicator of the number of ticks in nature from the results of entomological monitoring

Figure 7 shows the quantity of tick, taken from people.

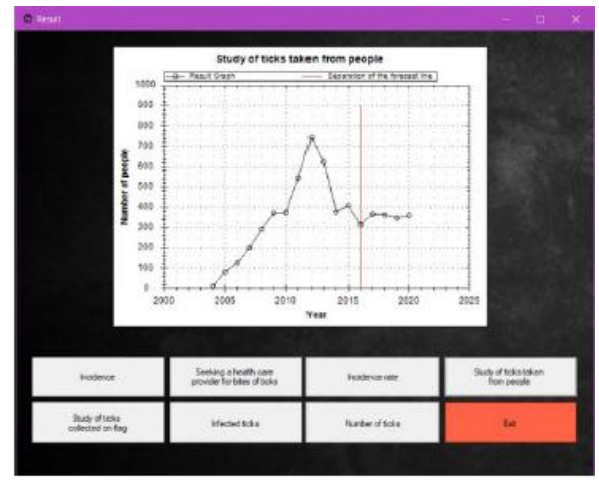

Figure7.Quantity of ticks taken from people

Figure 8 shows the number of ticks removed from the flag, i.e. with a special analysis of the study area.

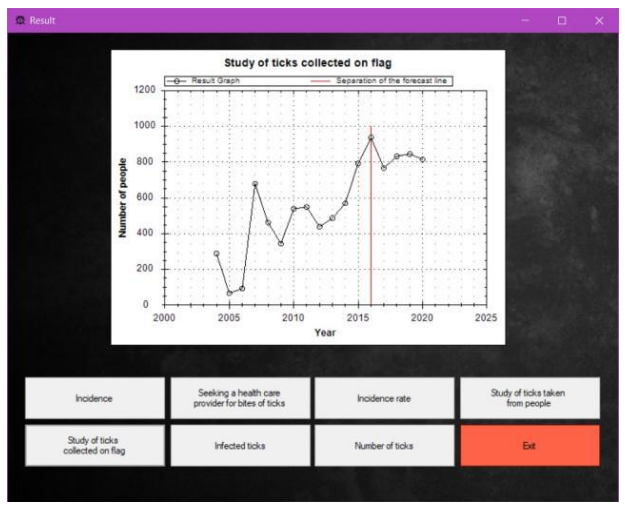

Figure8. Quantity of tick taken on flag

As can be seen from Figure 9, there is a lack of a direct relationship between the level of infection by Borrelias in ticks in nature and ticks removed from humans.

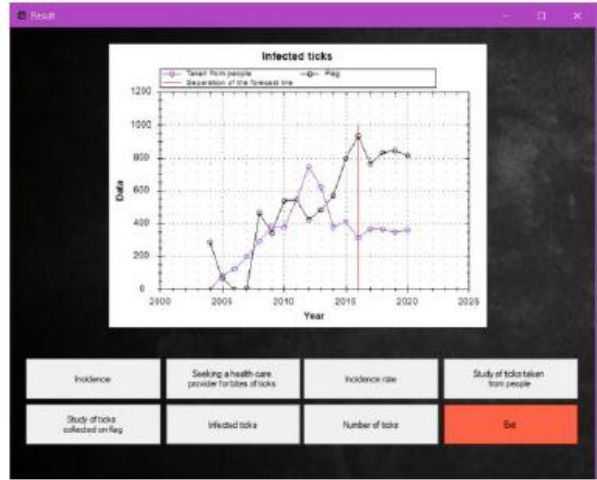

Figure9.Level of infection of ticks by Borrelia

\subsection{Estimation of the accuracy of the constructed forecast}

A comparative analysis of the accuracy of forecasting by moving average method to 3 years [10] and multiagent simulation showed that the latter describes the existing data better (Figure 10), therefore, the forecast will be more accurate.

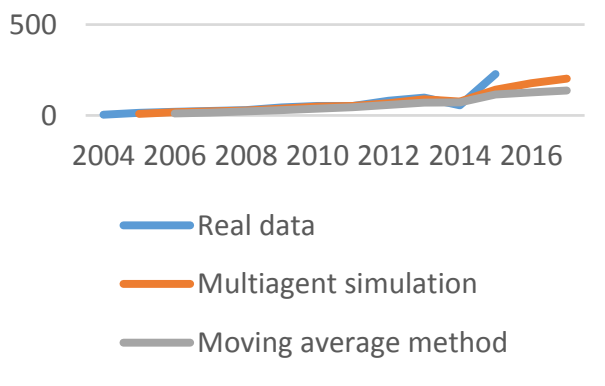

Figure10. Predicting the incidence by Ixodes tick borreliosis using different approaches

\section{Conclusions}

A comparative assessment of the accuracy of predicting the dynamics of the epidemic process using the moving average method and the multiagent approach was carried out. A higher accuracy is noted with the use of multi-agent simulation, on the basis of which a software package has been developed, which makes it possible to calculate the expected morbidity rate of the Ixodes tick borreliosis. The adequacy of the checked prognosis was verified on real statistical data on the incidence of Ixodes tick borreliosis in the Kharkiv region (Ukraine) from 2006 to 2016.

The obtained forecast revealed the expected persistence of the unstable epidemic situation with respect to the Ixodes tick borreliosis, which dictates the need to develop a set of preventive measures aimed at reducing the morbidity of people by Ixodes tick borreliosis. Virtual verification of the effectiveness of such events will be the next step in our study. 


\section{REFERENCES}

[1] Lindgren E., Jaenson T.G.T. Lyme borreliosis in Europe: influences of climate and climate change, epidemiology, ecology and adaptation measures // World Health Organization, 2006. - 35 p.

[2] Piletskiy P.E., Chumachenko D.I. Forecasting of incidence of Ixodes Tick Borreliosis by moving average method // XII International Pirogov scientific medical conference of students and young scientists. Book of abstracts. - M., 2017. pp. 184-185.

[3] Chumachenko D., Chumachenko T. Agent-Based Model of the Epidemic Process of Diseases with Multiple Routes of Infection Transmission Development and Evaluation // International Journal of Research Studies in Computer Science and Engineering (IJRSCSE), Volume 3, Issue 5. - India: ARC Publications Private Limited, 2016. - pp. 20-24.

[4] Chumachenko D., Yakovlev S. Investigation of agent-based simulation of malicious software // ECONTECHMOD. An international quarterly journal, Vol. 5, No 4. - Poland: Lublin-Rzeszow, 2016. - pp. 61-67.

[5] Yarovaya O.V., Chumachenko D.I. Programming and numerical methods. Part 2: Numerical methods using MatLab and Mathcad: guidance manual for laboratory works. - Kharkiv: National Aerospace University «KhAI», 2017. - 92 p.
[6] Chumachenko D., Chumachenko T. Agent-based investigation of sexually transmitted infection // Online Journal of Public Health Informatics, 9(1): e60, 2017.

[7] Chumachenko D.I. Intelligent simulation of the epidemic process of diphtheria infection // Internet and Society: Proceedings of VIII International scientific and practical conference, 7-8 July, 2017, Kutaisi, Georgia. - Kutaisi, 2017. - pp. $85-88$.

[8] Chumachenko D., Dobriak V., Mazorchuk M., Meniailov I., Bazilevych K. On agent-based approach to influenza and acute respiratory virus infection simulation // Proceedings of 14th International Conference on Advanced Trends in Radioelectronics, Telecommunications and Computer Engineering (TCSET), Lviv-Slavske, Ukraine, February 20-24, 2018.- paper 184.-4p.

[9] Акимов И.А., Небогаткин И.В. Иксодовые клещи городский ландшафтов г. Киева. Монография - Киев : Институт зоологии им. И.И.Шмальгаузена НАН Украины, 2016. -156 c.

[10] Piletskiy P.E., Chumachenko D.I. Moving average method in epidemic process forecasting //Advanced information systems and technologies: Proceedings of the V International scientific conference (May 17-19, 2017, Sumy). - Sumy: Sumy State University, 20

Citation: Dmytro Chumachenko, Pavlo Piletskiy, Mariia Sukhorukova. Intelligent Multiagent Approach to Morbidity by Ixodes Tick-Born Borreliosis Forecasting.ARC Journal of Public Health and Community Medicine.2018; 3(2):1-6. DOI: dx.doi.org/ 10.20431/2456-0596.0302001

Copyright: (C) 2018 Authors. This is an open-access article distributed under the terms of the Creative Commons Attribution License, which permits unrestricted use, distribution, and reproduction in any medium, provided the original author and source are credited. 